\title{
Transverse coupling impedance of the storage ring at the European Synchrotron Radiation Facility
}

\author{
T. F. Günzel* \\ European Synchrotron Radiation Facility, BP 220, 38043 Grenoble Cedex, France
}

(Received 6 June 2006; published 27 November 2006)

\begin{abstract}
The vertical and horizontal impedance budgets of the European Synchrotron Radiation Facility (ESRF) storage ring are calculated by element-by-element wake potential calculation. Resistive wall wakes are calculated analytically; the short range geometrical wakes are calculated by a 3D electromagnetic field solver. The effect of the quadrupolar wakes due to the flatness of most ESRF vacuum chambers is included in the model. It can well explain the sensitivity of the horizontal single bunch threshold on vacuum chamber changes, in particular, in low-gap sections of the ESRF storage ring. The values of the current thresholds on the transverse planes could be predicted correctly by the model within a factor of 2 .
\end{abstract}

DOI: 10.1103/PhysRevSTAB.9.114402

PACS numbers: 29.20.Dh

\section{INTRODUCTION}

The European Synchrotron Radiation Facility (ESRF) is a third-generation synchrotron light source operated by an electron accelerator complex consisting of a $172 \mathrm{MeV}$ LINAC, a $6 \mathrm{GeV}$ booster, and a storage ring of $844 \mathrm{~m}$ in circumference. The machine has been successfully in user operation since 1993. The storage ring produces photons mainly in the hard x-ray regime (critical energy $21 \mathrm{keV}$ ) with a brilliance of several $10^{20}$ photons $/ \mathrm{s} / \mathrm{mm}^{2} / \mathrm{mrad}^{2}$. The storage ring operates with a number of different time structures, namely, uniform filling, two-third filling, 16bunch filling, hybrid filling (two-third filling with a single bunch in the gap), and $8 \times 24$ filling. In order to maintain and improve its performance with regard to the demands of the users, all beam parameters are monitored and revised continuously. The main parameters of the ring are given in Table I.

\section{A. Impedance as an important issue in storage ring performance}

In the ESRF storage ring many effects contribute strongly to the coupling impedance and its associated effects such as tune shifts and instability thresholds. The storage ring has a large circumference and the majority of the vacuum chambers are made of stainless steel. Furthermore, the ring is equipped with a large number of low-gap sections to make it possible for the insertion devices (ID) to approach very closely to the beam. The low-gap ID sections contribute essentially to the resistive wall impedance as well as to geometrical impedance since transitions (cross section variations) to the wider cross section of the standard vacuum chamber are necessary. Over a large part of the circumference, either the vertical or the horizontal $\beta$-function assumes values of $35 \mathrm{~m}$ and

\footnotetext{
*Now working for CELLS, Apartado de Correos 68, E-08193 Bellaterra, Spain.

Electronic address: tguenzel@cells.es
}

more (Fig. 1). This behavior of the optical functions of the ESRF amplifies sensitively the tune shifts created by the transverse impedance. Furthermore, the momentum compaction factor $\alpha\left(1.86 \times 10^{-4}\right)$ is particularly small. This enhances the detuning of the transverse single bunch modes with current and makes them couple more easily. Indeed, the installation of more and more low-gap chambers in the straight sections of the storage ring deteriorated the operation conditions, in particular, in single bunch, of the machine. The single bunch thresholds, in particular, the horizontal one, at zero chromaticity decreased to very low values $(0.65 \mathrm{~mA}$ vertically and $1.7 \mathrm{~mA}$ horizontally in 2004). Moreover, incoherent tune shifts strongly move the working point, even up onto a quadrupolar resonance [1]. The compromised operation conditions required a thorough investigation of the sources of the transverse impedance, which is the main subject of this article. A campaign was started in order to localize the elements with the highest geometrical impedance by numerical elementby-element calculation. The use of 2D programs was in the majority of cases obsolete because the cross section of the vacuum chamber is mainly flat (Fig. 2), especially in the numerous low-gap sections (Fig. 3). Such chambers can no longer be assimilated to chambers of axial-symmetric cross section, especially because the cutoff frequency of round

TABLE I. ESRF machine parameters.

\begin{tabular}{lc}
\hline \hline \multicolumn{1}{c}{ Parameter } & Value \\
\hline Energy $(\mathrm{GeV})$ & 6 \\
Circumference $(\mathrm{m})$ & 844 \\
Beam current in multibunch mode (mA) & 200 \\
Natural energy spread, rms & $1 \times 10^{-3}$ \\
Natural bunch length, rms (ps) & 17 \\
Revolution frequency $\mu \mathrm{s}$ & 2.8 \\
Momentum compaction factor & $1.86 \times 10^{-4}$ \\
Synchrotron frequency at $8 \mathrm{MV}$ & $1.96 \mathrm{kHz}$ \\
Betatron tunes vertical/horizontal & $14.39 / 36.44$ \\
\hline \hline
\end{tabular}




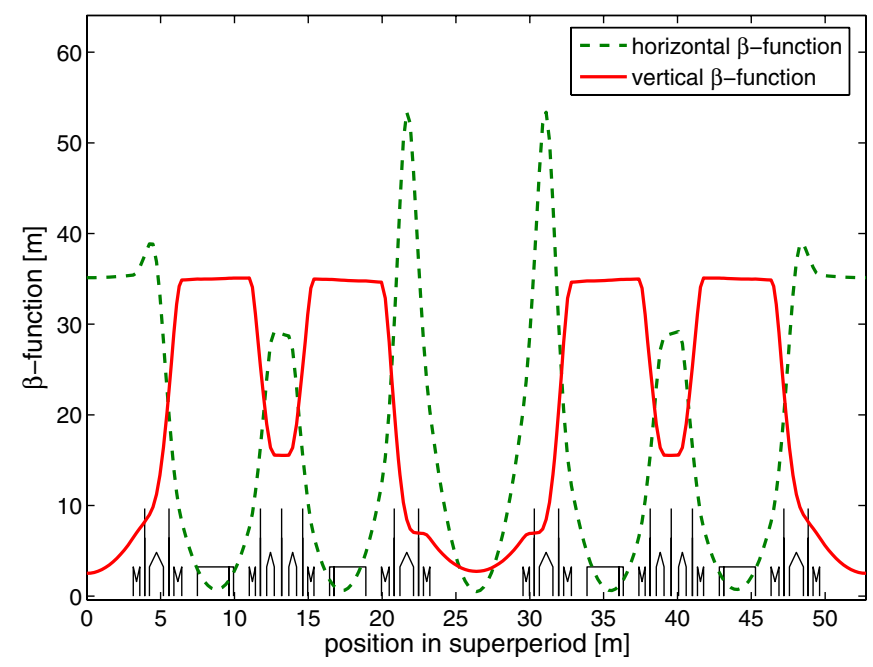

FIG. 1. (Color) The horizontal and vertical beta functions in one superperiod of the ESRF storage ring optics.

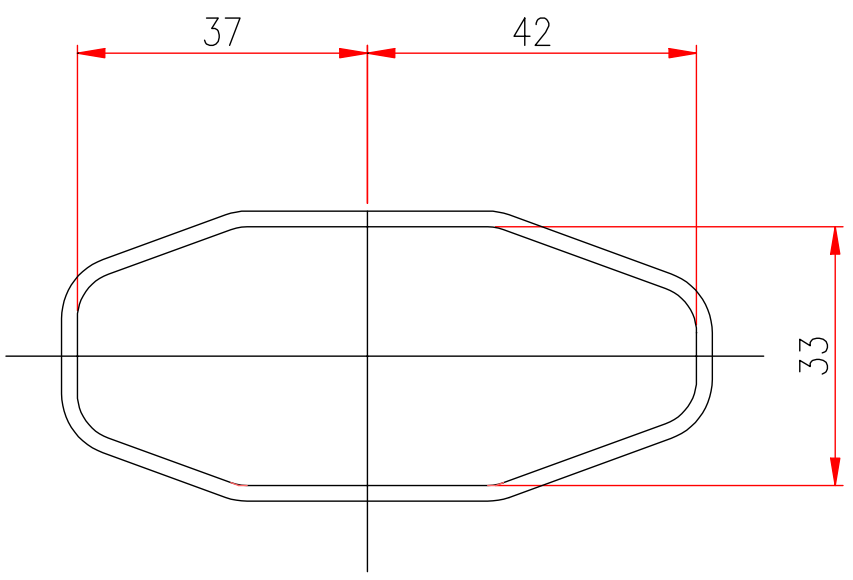

FIG. 2. (Color) Cross section of the standard vacuum chamber of the storage ring.

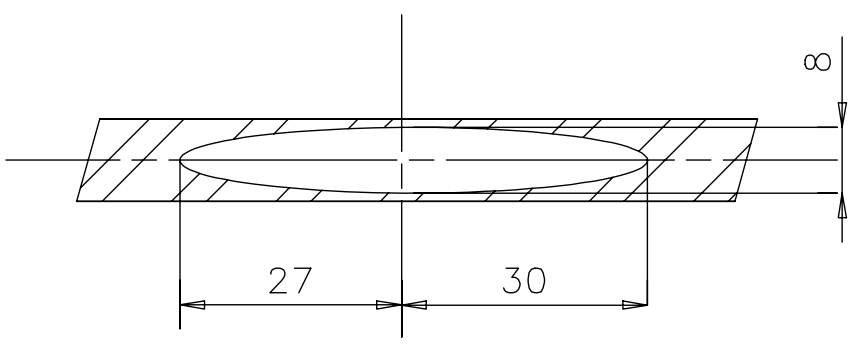

FIG. 3. Cross section of a $5 \mathrm{~m}$ long chamber of an aperture of $8 \mathrm{~mm}$ in an ID section.

and flat chambers is not the same. Consequently, the wake potential calculations were carried out with a 3D code (GDFIDL [2]). Another consequence of the flat geometry of the vacuum chamber is that the decomposition of the wake potential in axial modes of $\cos (m \theta)$ is no longer useful. However, as the ESRF uses an electron beam of
$6 \mathrm{GeV}$, it can be assumed that the beam moves at the speed of light. The longitudinal wake potential therefore fulfills the two-dimensional Laplace equation inside the beam tube. This allows for the existence of a quadrupolar wake potential (also called detuning wake) which only depends on the position of the test particle. This wake potential gives rise to an important current dependent tune shift which is defocusing on the vertical, and focusing on the horizontal plane. Despite the fact that this effect was already predicted in a series of papers [3-7] it is still not common knowledge. As this paper focuses mainly on impedance and less on beam dynamics related aspects, only simulations and predictions related to the transverse mode coupled instability (TMCI) will be presented. The threshold of the TMCI is used to validate the impedance model. In the conclusion of this paper the limits of this approach will be discussed. The paper is organized as follows. In Sec. II the existence of the quadrupolar wake is derived. In Sec. III the resistive wall impedance budget of both planes will be presented. This is followed in Sec. IV by the geometrical impedance budget. In Sec. V the coherent tune shifts and the TMC-instability thresholds are calculated on the base of the obtained transverse impedance budget. In Sec. VI the incoherent tune shifts will be calculated and added to the already obtained coherent tune shifts. In Sec. VII the resulting total tune shifts and the thresholds of the TMCI will be compared to experimental measurements at zero chromaticity.

\section{THE QUADRUPOLAR WAKE}

The quadrupolar wake responsible for the current dependent incoherent tune shift in both planes is first presented since it is fundamental for the following. The section closely follows Ref. [4]. Its existence in nonaxial symmetric chambers can be shown from first principles. We recall the definition of the longitudinal wake potential. For an exciting charge $q$ traveling with an offset $\left(x_{0}, y_{0}\right)$ from the beam axis, the longitudinal wake at distance $s$ behind the charge at the position $(x, y)$ is given by sampling and adding up the longitudinal electric field component $E_{z}$ :

$$
W_{l}\left(x, y, x_{0}, y_{0}, s\right)=-\frac{1}{q} \int_{-\infty}^{\infty} d z E_{z}\left(x, y, z, t=\frac{s+z}{c}\right) .
$$

As long as no transverse effects are involved, the dependence of the position of the exciting particle can be neglected. If the beam moves closely at the speed of light the longitudinal wake fulfills the Laplace equation with $r_{\perp}=$ $(x, y)$, respectively $r_{\perp}=(r, \theta)$ :

$$
\Delta_{\perp} W_{l}\left(s, r_{\perp}\right)=0 .
$$

Depending on the symmetry of the problem, this equation can be solved in polar or in Cartesian coordinates. The solution in polar coordinates is well known (the terms $r^{-m} m>0$ can be rejected because they are singular at 
$r=0)$ and given in most standard textbooks [8]:

$$
W_{l}(s, r, \theta)=\sum_{m} f_{m}(s) r^{m} \cos (m \theta) .
$$

In the case of a more general geometry, it is more useful to represent the solution in Cartesian coordinates:

$$
\begin{aligned}
W_{l}(s, x, y)= & C(s)+B(s) x+D(s) y+E(s)\left(y^{2}-x^{2}\right) \\
& +F(s) x y+O\left(x^{3}, y^{3}\right) .
\end{aligned}
$$

Considering that the exciting and test particle are located close to the beam axis, the higher order terms $O\left(x^{3}\right)$ are very small and can be neglected. Furthermore, if the chamber geometry presents a plane symmetry the longitudinal wake must fulfill one of the following symmetry conditions (which only remains valid as long as the exciting particle is on axis):

$$
\begin{array}{ll}
W_{l}(x, y)=W_{l}(-x, y) \rightarrow F(s)=0 & B(s)=0 \\
W_{l}(x, y)=W_{l}(x,-y) \rightarrow F(s)=0 & D(s)=0 .
\end{array}
$$

The standard vacuum chamber of the ESRF ring is symmetrical on the vertical plane (Fig. 2). Furthermore, the horizontal symmetry is only slightly broken, so if the initial vertical symmetry is broken by an intrusion the quasisymmetry on the horizontal plane keeps the skew term $F(s) x y$ small. Figure 6 shows a typical longitudinal wake potential at the middle of the bunch $(s=0.03 \mathrm{~m}$ for a $5 \mathrm{~mm}$ long bunch) in the vacuum chamber of a low-gap section. The term $x^{2}-y^{2}$ can be recognized from the saddle point form of the contour. However, if the symmetry on both planes is broken by an important horizontal and vertical intrusion $F(s) x y$ can be significantly nonzero. There are only a very few chamber geometries in the ESRF ring where this is the case. If an offset to the exciting particle is applied, the symmetry on the corresponding plane is broken and the corresponding coefficient $B(s)$ or $D(s)$ becomes nonzero. For a vertical offset of the exciting particle $y_{0} W_{l}$ becomes (neglecting the skew term) [9]

$$
\begin{aligned}
W_{l}\left(s, x, y, y_{0}\right)= & C(s)+B(s) x+D(s) y+A(s) y y_{0} \\
& +E(s)\left(y^{2}-x^{2}\right)+O\left(x^{3}, y^{3}\right) .
\end{aligned}
$$

Using the Panofsky-Wenzel theorem [10]

$$
W_{(x, y)}(s, x, y)=-\int_{0}^{s} \nabla_{(x, y)} W_{l}(s, x, y) d s^{\prime},
$$

the transverse wake potentials $W_{x}\left(s, x, y, y_{0}\right)$ and $W_{y}\left(s, x, y, y_{0}\right)$ can be derived from the longitudinal wake potential. Lower case coefficients are used which are linked to the uppercase coefficients by $a(s)=$ - $\int_{0}^{s} A\left(s^{\prime}\right) d s^{\prime}$ etc.:

$$
\begin{aligned}
& W_{x}\left(s, x, y, y_{0}\right)=b(s)-2 e(s) x \\
& W_{y}\left(s, x, y, y_{0}\right)=d(s)+a(s) y_{0}+2 e(s) y .
\end{aligned}
$$

The transverse wake can be decomposed into a monopolar part (only depending on the longitudinal coordinate $s$ ), a dipolar part (depending on the source particle offset $y_{0}$ and $s$ ), and a quadrupolar field. The quadrupolar field which only depends on the test particle coordinates $(x, y, s)$, however, contributes to the incoherent tune shift. The found behavior is general, i.e., it is not limited to a special type of wake potential. As the strength of the quadrupolar field $e(s)$ is a function of $s$, it varies along the longitudinal charge distribution of a bunch which gives rise of a spread of the betatron tune.

As they are needed later, the definitions of transverse wakefield and transverse impedance are recalled. The notation $W_{\perp}$ as it is used in the literature denotes the first, respectively second, component of the vector:

$$
\mathbf{W}_{\perp}=\left(\frac{\partial W_{x}\left(s, x, y, x_{0}, y_{0}\right)}{\partial x_{0}}, \frac{\partial W_{y}\left(s, x, y, x_{0}, y_{0}\right)}{\partial y_{0}}\right) .
$$

Assuming that the wakes of the integrands are created by a pointlike charge, the horizontal and vertical impedance as a function of $\omega$ representing the angular frequency are defined by

$$
\begin{aligned}
& Z_{H}(\omega)=\frac{i}{c} \int_{-\infty}^{\infty} \frac{\partial W_{x}\left(s, x, y, x_{0}, y_{0}\right)}{\partial x_{0}} e^{-i \omega s / c} d s \\
& Z_{V}(\omega)=\frac{i}{c} \int_{-\infty}^{\infty} \frac{\partial W_{y}\left(s, x, y, x_{0}, y_{0}\right)}{\partial y_{0}} e^{-i \omega s / c} d s .
\end{aligned}
$$

Furthermore, the notion of quadrupolar impedance is introduced:

$$
\begin{aligned}
Z_{H}^{\text {quad }} & =\frac{i}{c} \int_{-\infty}^{\infty} \frac{\partial W_{x}\left(s, x, y, x_{0}, y_{0}\right)}{\partial x} e^{-i \omega s / c} d s \\
Z_{V}^{\text {quad }} & =\frac{i}{c} \int_{-\infty}^{\infty} \frac{\partial W_{y}\left(s, x, y, x_{0}, y_{0}\right)}{\partial y} e^{-i \omega s / c} d s .
\end{aligned}
$$

Noting that $Z_{V}^{\text {quad }}=-Z_{H}^{\text {quad }}$ henceforth the simplified notation $Z^{\text {quad }}=Z_{V}^{\text {quad }}$ will be used. Furthermore, for later use the definition of the transverse kick factor is given (using $\sigma_{\tau}$ as bunch length in times units):

$$
\kappa_{\perp}\left(\sigma_{\tau}\right)=\frac{1}{2 \pi} \int_{-\infty}^{\infty} \operatorname{Im} Z_{\perp}(\omega) \exp \left(-\omega^{2} \sigma_{\tau}^{2}\right) d \omega .
$$

The definition of effective impedance differs only by a factor of $2 \sqrt{\pi} \sigma_{\tau}$ :

$$
Z_{\perp}^{\mathrm{eff}}\left(\sigma_{\tau}\right)=\frac{\sigma_{\tau}}{\sqrt{\pi}} \int_{-\infty}^{\infty} \operatorname{Im} Z_{\perp}(\omega) \exp \left(-\omega^{2} \sigma_{\tau}^{2}\right) d \omega .
$$

Henceforth, if not explicitly stated differently, the sign $\perp$ (in $Z_{\perp}, Z_{\perp}^{\text {eff }}$ and $\kappa_{\perp}$ ) is not only used as a symbol for $H$ (horizontal) or $V$ (vertical), but also for quad (quadrupolar component) in order to generalize the definition of the transverse kick factor and effective impedance. This allows one to treat both coherent and incoherent mode detuning in 
an equivalent way. But in fact the generalized definition is only used in Sec. VI. Finally, preference was given to a distinction of the impedance of the transverse planes by $H$ and $V$ instead of $x$ and $y$ to avoid later conflicts in notation.

\section{RESISTIVE WALL IMPEDANCE}

In this study the resistive wall impedance is important in two respects. First, it shows very well the role of the quadrupolar wake in impedance related issues. Second, its contribution to the total impedance budget is significant. In the whole study only the low frequency range $\omega \ll c / s_{0}$ $\left(\sim 10 \mathrm{THz}\right.$ in the case of the ESRF) is considered where $s_{0}$ represents $\left(\frac{b^{2}}{Z_{0} \sigma}\right)^{1 / 3}, Z_{0}$ the free space impedance, $\sigma$ the electrical conductivity of the wall material, and $b$ the half of the vertical beam pipe extension.

\section{A. Resistive wall wake potential decomposition}

In case of resistive wall impedance the behavior of the transverse wakes, especially the quadrupolar wake, was already studied by [11] for a beam pipe of rectangular and elliptical, by [7] for a beam pipe of general cross section. The main results from these publications are recalled now as they are very useful to introduce the subject. The different components of the transverse resistive wall wake are graphically represented in Fig. 4. The formal expressions necessary for the generation of this graph were taken from another closely related publication [12].

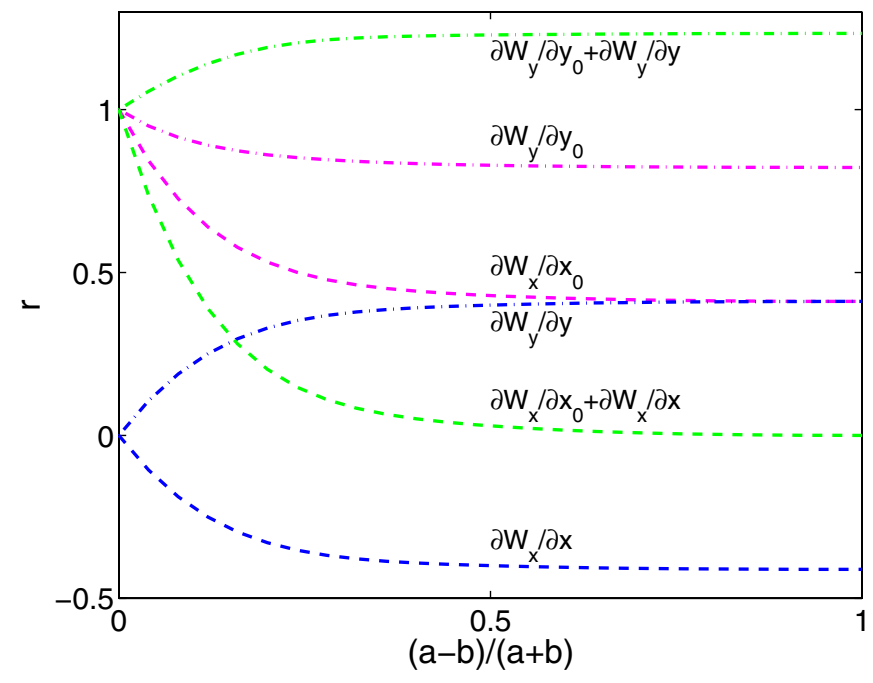

FIG. 4. (Color) $r$ represents the ratio of transverse resistive wall wake potentials for elliptic pipes of horizontal extension $2 a$ and vertical extension $2 b$ to those of a round pipe of radius $b$ as function of $q=\frac{a-b}{a+b}$ at large $s \gg s_{0}$. $W_{y}$ denotes the vertical, $W_{x}$ the horizontal wake, $y_{0}$ the vertical position of the source particle, $y$ the vertical position of the test particle, $x_{0}$ the horizontal offset of the source particle, and $x$ the horizontal offset of the test particle. $q=0$ represents a round pipe, $q=1 \mathrm{a}$ flat pipe.
If the vacuum chamber is round, the horizontal and vertical dipolar wake are identical, whereas the quadrupolar wake components are zero. If the chamber undergoes a transition to an elliptical form, in the extreme case up to two horizontal parallel plates, the vertical dipolar wake becomes smaller and assumes asymptotically the value of $\frac{\pi^{2}}{12}$ (normalized to the value of the round chamber), whereas the horizontal dipolar wake decreases even stronger to half of that value, i.e. $\frac{\pi^{2}}{24}$. On the other hand, the quadrupolar wake components, zero for a round chamber value, both become nonzero, are of opposite sign, and their absolute value increases to the value of the horizontal dipolar wake, i.e. $\frac{\pi^{2}}{24}$. This result is resumed by the following expressions for the transverse impedance per unit length [with $\delta(\omega)=$ $\sqrt{(2 c) /\left(\sigma Z_{0}|\omega|\right)}$ as skin depth].

Circular cross section with radius $b$ :

$$
\begin{gathered}
Z_{V}=[\operatorname{sign}(\omega)+i] \frac{Z_{0}}{2 \pi b^{3}} \delta(\omega) \\
Z_{H}=[\operatorname{sign}(\omega)+i] \frac{Z_{0}}{2 \pi b^{3}} \delta(\omega) \\
Z^{\text {quad }}=0 .
\end{gathered}
$$

Parallel plate geometry ( $b$ is the half distance between the parallel plates):

$$
\begin{gathered}
Z_{V}=\frac{\pi^{2}}{12}[\operatorname{sign}(\omega)+i] \frac{Z_{0}}{2 \pi b^{3}} \delta(\omega) \\
Z_{H}=\frac{\pi^{2}}{24}[\operatorname{sign}(\omega)+i] \frac{Z_{0}}{2 \pi b^{3}} \delta(\omega) \\
Z^{\text {quad }}=\frac{\pi^{2}}{24}[\operatorname{sign}(\omega)+i] \frac{Z_{0}}{2 \pi b^{3}} \delta(\omega) \\
Z_{V}^{\text {quad }}=Z_{V} / 2 \quad Z_{H}^{\text {quad }}=-Z_{H} .
\end{gathered}
$$

All impedances depend only on one single geometrical parameter, the vertical half-extension, respectively radius, of the chamber $b$. If the test particle and the exciting particle have the same offset in a parallel plate geometry, the impedance $Z^{\text {comp }}$ composite of dipolar and quadrupolar parts can be written

$$
\begin{aligned}
Z_{V}^{\text {comp }}=Z_{V}+Z^{\text {quad }} & =\frac{\pi^{2}}{8}[\operatorname{sign}(\omega)+i] \frac{Z_{0}}{2 \pi b^{3}} \delta(\omega) \\
Z_{H}^{\text {comp }} & =Z_{H}-Z^{\text {quad }}=0 .
\end{aligned}
$$

If the exciting particle and test particle have the same offset, the test particle is subjected to an increased defocusing force in the vertical direction whereas it does not feel any force in the horizontal direction. However, this does not mean that the horizontal impedance $Z_{H}$ is zero, it is only compensated by the focusing force of the quad- 
TABLE II. Resistive wall budget vertical and horizontal plane. SS stands for stainless steel, NEG is explained in the text. All invacuum undulators are $2 \mathrm{~m}$ long except the prototype of $1.6 \mathrm{~m}$ length (therefore a fractional quantity is written). The first line is only given for information and not taken into account in the budget.

\begin{tabular}{|c|c|c|c|c|c|c|c|c|}
\hline Chamber type & Material & Length $[\mathrm{m}]$ & Half-gap [mm] & Quantity & $\beta_{V}[\mathrm{~m}]$ & $\beta_{H}[\mathrm{~m}]$ & $(\beta Z)_{V}^{\mathrm{eff}}[\mathrm{M} \Omega]$ & $(\beta Z)_{H}^{\mathrm{eff}}[\mathrm{M} \Omega]$ \\
\hline In-vacuum undulators (closed) & $\mathrm{Cu}$ & 2 & 3 & 7.8 & 3.53 & 8.75 & 0.138 & 0.171 \\
\hline In-vacuum undulators (open) & $\mathrm{Cu}$ & 2 & 15 & 7.8 & 3.53 & 8.75 & 0.001 & 0.001 \\
\hline Low-gap chamber & SS & 5 & 5.5 & 8 & 3.4 & 20.0 & 0.372 & 1.091 \\
\hline Low-gap chamber $1 \mu \mathrm{m}$ NEG & $\mathrm{Al} / \mathrm{NEG}$ & 5 & 5.5 & 2 & 3.64 & 35.19 & 0.029 & 0.143 \\
\hline Low-gap chamber $1 \mu \mathrm{m} \mathrm{NEG}$ & $\mathrm{SS} / \mathrm{Cu} / \mathrm{NEG}$ & 5 & 4 & 1 & 3.51 & 35.19 & 0.032 & 0.164 \\
\hline Low-gap chamber $1 \mu \mathrm{m} \mathrm{NEG}$ & $\mathrm{Al} / \mathrm{NEG}$ & 5 & 4 & 6 & 3.36 & 25.06 & 0.214 & 0.795 \\
\hline Low-gap chamber $0.5 \mu \mathrm{m}$ NEG & $\mathrm{Al} / \mathrm{NEG}$ & 5 & 4 & 3 & 3.36 & 35.19 & 0.087 & 0.462 \\
\hline Remaining elements & & & & & & & 0.133 & 0.218 \\
\hline Standard storage ring chamber & SS & 672 & 16.5 & 1 & 24.6 & 16.6 & 1.643 & 0.555 \\
\hline Total (in-vacuum open) & & & & & & & 2.511 & 3.429 \\
\hline
\end{tabular}

rupolar field expressed by $Z^{\text {quad }}$. Finally henceforth the ratios of the resistive wall wakes as they are represented in Fig. 4 are referred to as form factors.

\section{B. Resistive wall budget}

For the time being, there is no numerical code available which calculates a wake from an arbitrary geometry including the resistive wall contribution. For this reason, the resistive wall budget of the vertical and horizontal dipolar components of the wake has to be computed separately from the numerical evaluation of the geometrical impedance. The vacuum chambers are considered as two parallel plates. This is justified because, even for chambers which are not completely flat, the form factors are already very close to the values of two parallel plates. So apart from the rf cavities which are of round cross section, the form factors for flat chambers are applied to the standard resistive wall impedances. In addition, each contribution is weighted by the local $\beta$-function of the corresponding element. The overview of the resistive wall budget can be found in the Table II. The walls of nonevaporable getter (NEG)-coated vacuum chambers in the storage ring were considered as a double layer of $1 \mu \mathrm{m}$, respectively $0.5 \mu \mathrm{m}, \mathrm{NEG}$ (nonevaporable getter out of $\mathrm{Ti} / \mathrm{Zr} / \mathrm{V}$ ) and the underlying metal ( $1 \mathrm{~mm}$ aluminum or $35 \mu \mathrm{m}$ copper). Reference [13] showed that a NEG layer mainly increases the imaginary part of the transverse impedance and does not affect the real part. However, in order to adopt a simplified model the $\omega^{-0.5}$ behavior of the resistive wall impedance was maintained (both for the real and the imaginary part) but scaled in order to provide the correct kick factor of the double layer (Fig. 5). This method overestimates the real part of the transverse impedance, but reproduces fairly accurately the imaginary part which is responsible for the tune shift. The double layer model seems to be a fair representation of NEG-coated aluminum chambers[14]. A large discrepancy was, however, found between the measured and calculated impedances for the
NEG-coated copper-covered stainless steel chambers [14]. It is very well possible that the model used here of a double layer cannot be applied for the three layer case (NEG/Cu/stainless steel).

The in-vacuum undulators are not of primary importance in the budget because, apart from one, all are in odd-numbered cells with moderate horizontal and low vertical $\beta$-functions. Furthermore, they are 2.5 (the prototype even 3.125) times shorter than standard low-gap chambers and are inside equipped with a $\mathrm{CuNi}$-sheet providing good conductivity. Their contribution to the total resistive wall budget increases by about $5 \%$ if they are closed to a gap of $6 \mathrm{~mm}$. In the table, the values for the open undulators are also quoted, because in Sec. VII they are compared to results of threshold measurements which were only possible under this condition.

The largest contribution to the $\beta$-weighted vertical resistive wall impedance is the standard storage ring vacuum chamber (Fig. 2), because the vertical $\beta$-function assumes its highest values outside of the low-gap sections. On the

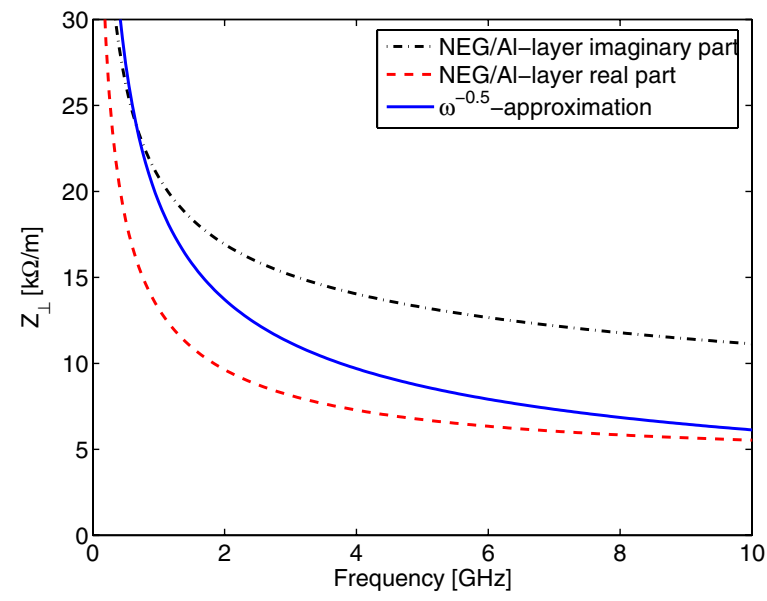

FIG. 5. (Color) Comparison of the $\omega^{-0.5}$ approximation with the true imaginary and real part of the resistive wall impedance of a $\mathrm{NEG} / \mathrm{Al}$ double layer. 
other hand, since in the low-gap sections the values of the horizontal $\beta$-function are much higher than the vertical ones (Fig. 1), the $\beta$-weighted transverse impedance of the low-gap sections in the horizontal plane is much higher than in the vertical plane (the smaller form factor in the horizontal plane cannot compensate for this effect). This is still the case after the addition of the $\beta$-weighted transverse impedance of the standard storage ring vacuum chamber outside of the low-gap sections. Consequently, contrary to the vertical, the horizontal resistive wall impedance budget is much more sensitive to the reduction of the vertical gaps by the installation of new vacuum chambers in the ID sections.

The transverse impedance of the kickers created by the ceramic chamber covered with a TiN layer surrounded by a ferrite was not taken into account. An estimation carried out with the formulas of [15] shows that even when varying the titanium resistivity of $\rho=7 \times 10^{-7} \Omega \mathrm{m}$ by a factor of 2 , respectively 0.5 , the $\beta$-weighted transverse impedance on both planes is not more than $160 \mathrm{k} \Omega$ above $1 \mathrm{GHz}$ and decreases strongly at higher frequencies. So the effect on the total budget is small, in particular, if the high-frequency part of the transverse impedance spectrum is considered. However, the variation of the shape of the kicker chamber is taken into account in the geometrical impedance budget.

\section{GEOMETRICAL IMPEDANCE}

In this section the calculation of the transverse geometrical impedance with the program GDFIDL is described. The decomposition of the wakes in dipolar and quadrupolar parts is illustrated. Finally, the budget of the geometrical impedance is established.

\section{A. GDFIDL simulations}

The 3D code GDFIDL [2] was chosen in order to establish the impedance budget of all elements of the ESRF ring caused by cross section variation. With GDFIDL running on several processors in parallel, the calculation of wake potentials in large geometries on a fine mesh is feasible. At the beginning of this study, results of GDFIDL were compared to those of ABCI [16] and MAFIA [17]. For taper pairs with a circular cross section the results of GDFIDL and $\mathrm{ABCI}$ agree up to a frequency of almost $20 \mathrm{GHz}$ [18]. In order to obtain the wakes inside the beam tube, the longitudinal electrical field is integrated along the beam tube wall. It is advantageous to use only convex structures for this type of calculations. This ensures that, at the entry and exit of the structure, the integration path for the longitudinal component of the electrical field is on the conducting material where this field component is zero. However, apart from cavitylike structures, most geometries, in particular, low-gap chambers equipped with tapers, are of concave form and cannot be dealt in this manner (keeping the integration path on the border of the exit tubes the path would traverse zones outside of the tube and becomes useless). The usual way [19] to overcome this problem is to invert the geometry, i.e., to exchange the larger outer beam tubes with the inner low-gap chamber. This is called the indirect method. The second possibility is to place the integration path inside of the entry and exit beam tube so that it remains inside along the whole structure (direct method). But in the latter case the result is exposed to larger numerical noise, and is therefore less precise. Most low-gap chambers were simulated by using the indirect method to avoid the noise. However, for structures with variable gap, i.e., different types of in-vacuum undulators, the direct method was applied. Furthermore, in taperlike structures the distance between the tapers was optimized. The distance was increased until the impedance no longer shows any significant signs of change. For the few cases where the direct method was used, the computed impedance at zero frequency was very close to the value computed by the indirect method. At higher frequencies larger deviations appear. The use of the indirect method generates a systematic error of the impedance calculation, most important at higher frequencies. The existence of the quadrupolar wake was checked with GDFIDL. An example is shown in Fig. 6. To obtain the impedance budget, the geometry of the storage ring was decomposed into different elements which were simulated independently. The results were added up linearly. However, this assumes that the different elements of the ring do not interact with each other which is a simplification. In particular, at the entry of low-gap ID chambers many different elements like pumps, beam position monitors (BPMs), shielded bellows (rf fingers), and tapers closely follow each other. Limited computing resources have not, up until now, made a simulation of such combinations possible.

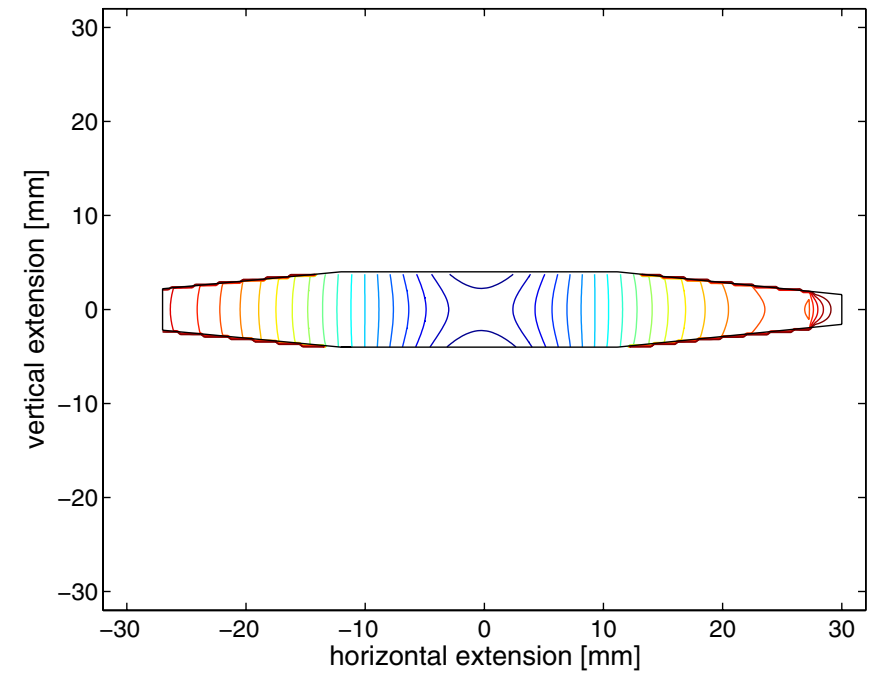

FIG. 6. (Color) Lines of constant values of $W_{l}(x, y)$ at the middle of the bunch $(s=0.03 \mathrm{~m})$ in a low-gap vacuum chamber. The colors indicate the different levels. The function $W_{l}(x, y)$ has a saddle point in the middle of chamber which demonstrates the local $x^{2}-y^{2}$ behavior. 


\section{B. Wake potential decomposition}

GDFIDL was also used to decompose the calculated wake potentials in the dipolar and the quadrupolar part. The different proportions of the total wake which are attributed to the dipolar and the quadrupolar wake after the decomposition are similar to those which are obtained in the case of the resistive wall. In the program it is possible to compute the transverse wake potentials for independent transverse offset of the source and test particle. As a result, both the dipolar and the quadrupolar wakes can be derived. Figure 7 represents a vertical wake computed for a taper geometry with a flat polygonal cross section. The magenta curve is the dipolar wake computed with the test particle undisplaced. The green curve is a wake computed with

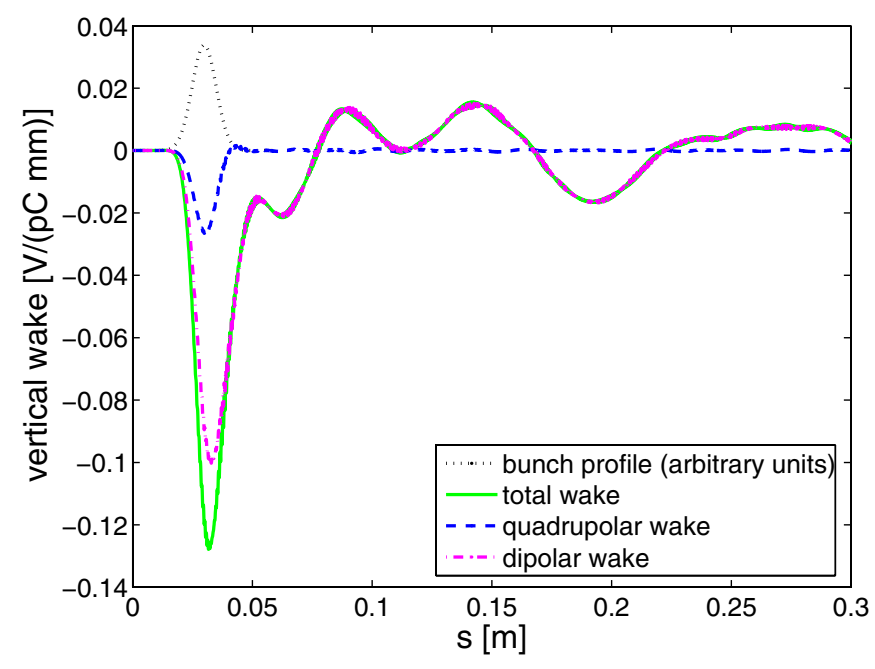

FIG. 7. (Color) Decomposition of the total vertical wake (green) in its dipolar part (magenta) and quadrupolar part (blue). The black dashed curve is the electron beam profile.

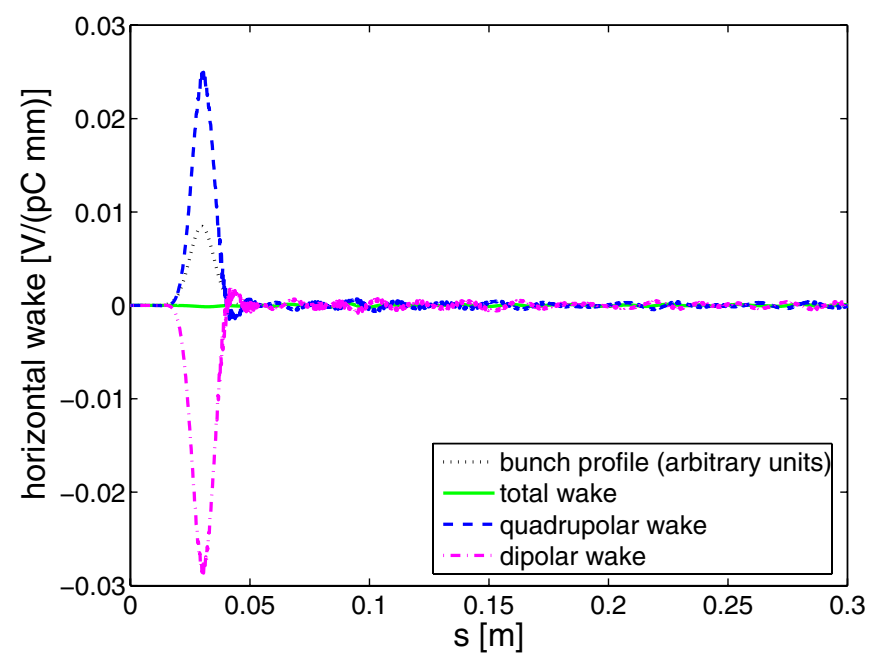

FIG. 8. (Color) Decomposition of the total horizontal wake (green) in its dipolar part (magenta) and quadrupolar part (blue). The black dashed curve is the electron beam profile.

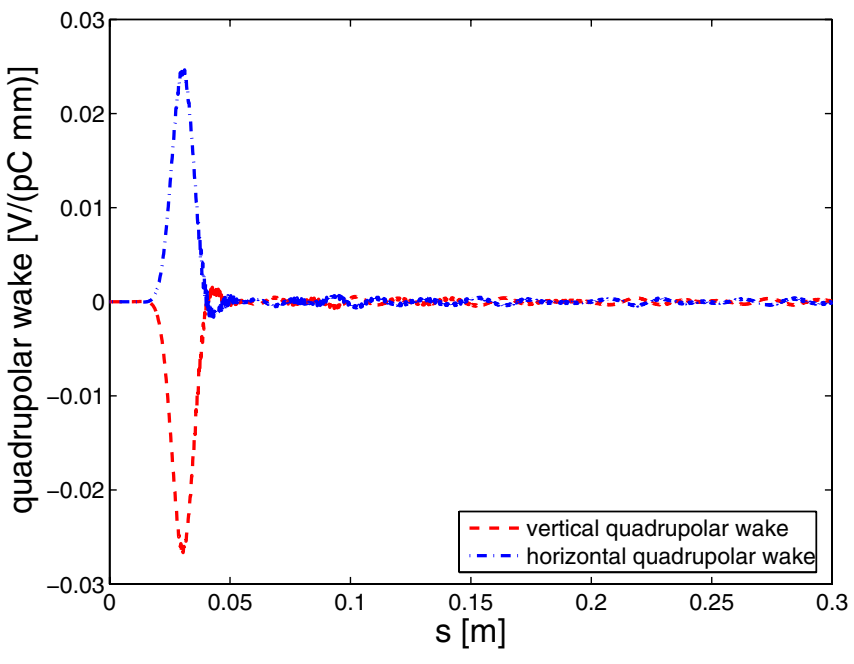

FIG. 9. (Color) The quadrupolar component of the vertical wake (red) and horizontal wake (blue).

both particles displaced. The difference between the green and the magenta curve is the quadrupolar wake drawn in blue in Fig. 7. The same procedure is applied in the horizontal plane whose result in shown in Fig. 8. The blue curve is the quadrupolar wake. As expected, the quadrupolar wakes computed in both planes are equal but of opposite sign as shown in Fig. 9. The described decomposition is always applied to separate the dipolar wakes from the quadrupolar wakes in both planes. This made it possible to determine both components of the wake for all simulated vacuum chamber elements.

Also existing in chambers without up-down or left-right symmetry is a nonzero monopolar wake $[b(s)$ and $d(s)$ in Eq. (8)]. In the wakefield decomposition it is also subtracted from the total wake. For simplicity reasons, this wake has been neglected in this paragraph.

\section{Budget}

For numerous elements of the ESRF-ring the dipolar and quadrupolar component of both transverse wakes were determined. A total of 1776 elements were taken into account, thereof 569 flanges, 448 horizontal pumps, 293 rf fingers, 277 BPMs, 134 vertical pumps, 27 transitions to standard low-gap chambers, 8 in-vacuum undulators, 6 cavities, 3 tapers to the cavities, 2 scrapers, and 9 other elements. The vertical and horizontal impedance of each element was calculated from the corresponding dipolar component. The quadrupolar component is later used for the calculation of the incoherent tune shift in Sec. VI. The impedances of the different elements were multiplied by their local $\beta$-function. The resulting spectra of the different types of elements are added up and shown in Figs. 10-13. The vertical impedance budget is created by many different elements, amongst which the shielded bellows and the flanges play a major role. In fact, the low-gap chambers only contribute $20 \%$ to the vertical budget. However, the 


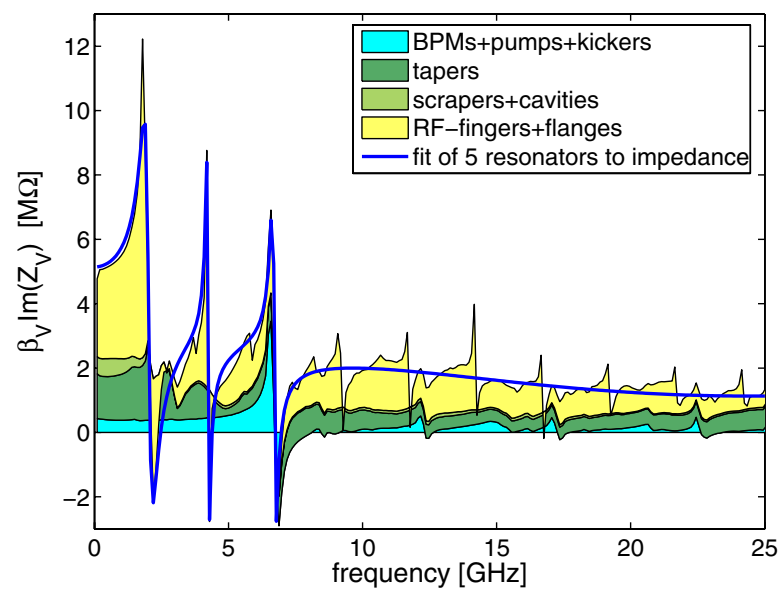

FIG. 10. (Color) Spectrum of the $\beta$-weighted imaginary part of the vertical impedance due to geometrical variation.

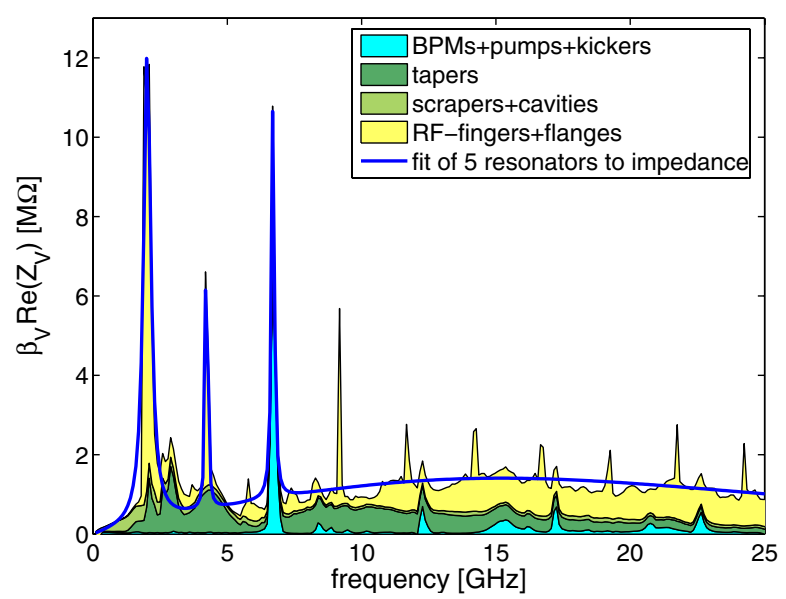

FIG. 11. (Color) Spectrum of the $\beta$-weighted real part of the vertical impedance due to geometrical variation.

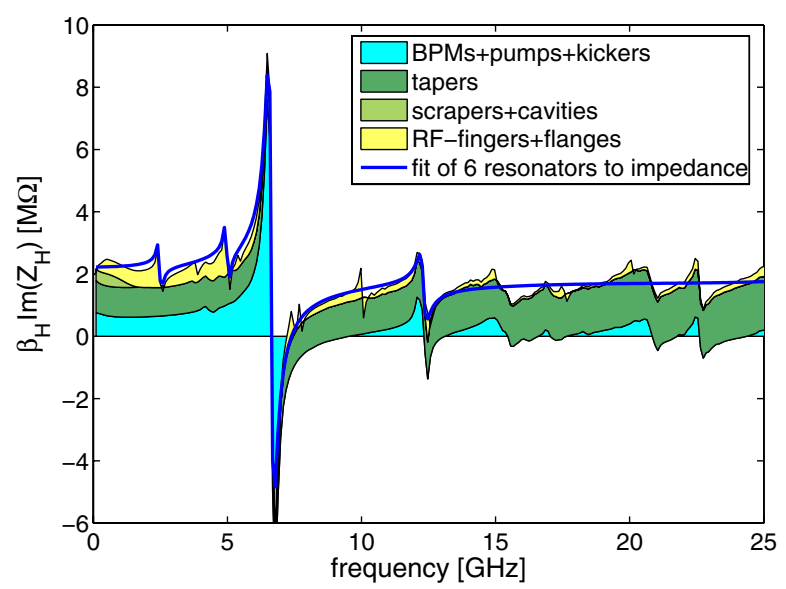

FIG. 12. (Color) Spectrum of the $\beta$-weighted imaginary part of the horizontal impedance due to geometrical variation.

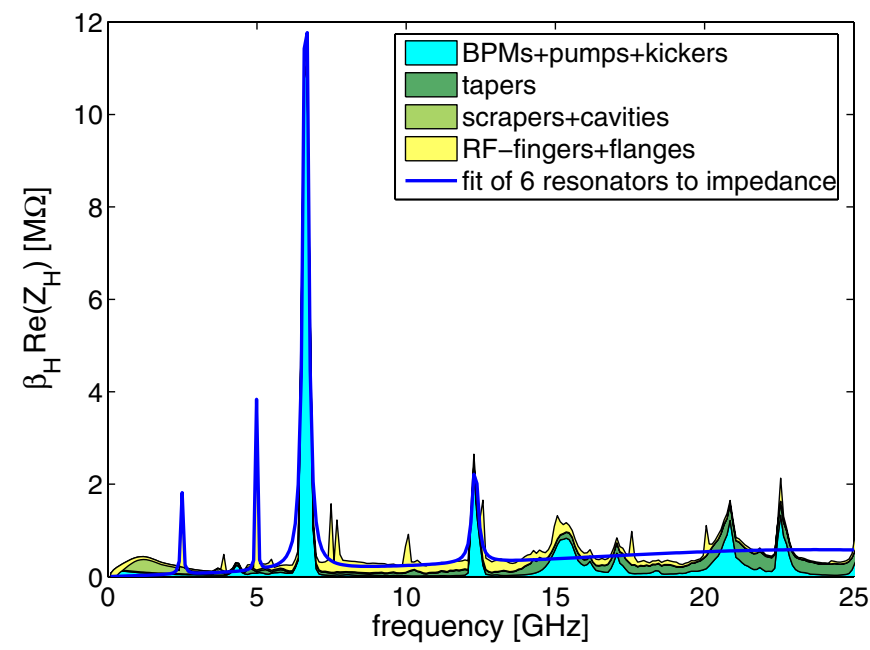

FIG. 13. (Color) Spectrum of the $\beta$-weighted real part of the horizontal impedance due to geometrical variation.

low-gap chambers make up a much larger part of the horizontal budget and dominate together with the BPMs. As in the case of the $\beta$-weighted resistive wall impedance budget, the low-gap chambers dominate more in the horizontal plane than in the vertical one. A composition of 5 (6) broadband resonators was fitted to the spectra in the vertical (horizontal) plane for further computation of the instability thresholds. Particular attention was paid to the vacuum chambers in the bending magnets. They can be considered as horizontal tapers. But the computed horizontal effective impedance of all bending magnet chambers only is $Z_{H}^{\text {eff }}=90 \frac{\mathrm{m} \Omega}{\mathrm{m}}$ for a $5 \mathrm{~mm}$ bunch length [20]. This confirms that the geometry variation on the horizontal plane has far less impact on the transverse impedance than a geometry variation on the vertical plane.

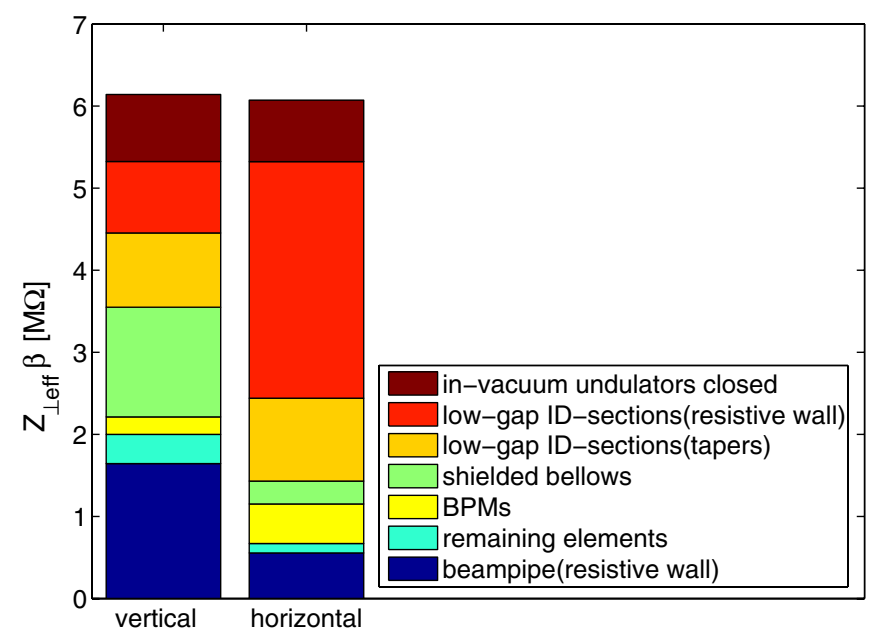

FIG. 14. (Color) Effective vertical and horizontal impedance budget weighted by the local $\beta$-function. Only the dipolar component is taken into account in the effective impedance. 
Combining the $\beta$-weighted geometrical impedance and resistive wall impedance, the $\beta$-weighted effective impedance for all elements was calculated in both planes (Fig. 14). It confirms the fact that the $\beta$-weighted vertical impedance is distributed all along the machine whereas the $\beta$-weighted horizontal impedance is dominated by the low-gap ID chambers and the BPMs. The $\beta$-function distribution along the ring is mainly responsible for this peculiar behavior. Closing all 8 in-vacuum undulators to a gap of $6 \mathrm{~mm}$ increases both transverse $\beta$-weighted impedances by about $12 \%$.

\section{TMCI THRESHOLDS}

In order to compute the mode coupling in single bunch at zero chromaticity, the parameters describing the different broadband resonators obtained from the spectral fits were entered into MOSES [21] together with a parametrization of the resistive wall impedance. The original source code of MOSES was modified for this purpose to accept multiple broadband resonators and resistive wall impedance. The status of the in-vacuum undulators was considered as open to allow for the comparison of the computation to the measurements of the tune shifts. Furthermore, a current dependent bunch length was assumed according to a simplified parametrization derived from bunch length measurements [22] $\sigma_{\tau}=\sigma_{\tau 0}(1+$ $I[\mathrm{~mA}] / 2.3)$ (with $I$ as single bunch current). In addition, it is assumed that the product of bunch length and incoherent synchrotron tune is current independent $\sigma_{\tau}(I) \nu_{s}(I)=$ const $[23,24]$. This assumption is reasonable as long as the single bunch current is below $2 \mathrm{~mA}$ where the bunch length is still in the potential well regime (but it has neither been experimentally checked nor cross-checked with the distortion of the potential well). As a consequence, the incoherent synchrotron tune becomes current dependent. The mode coupling calculated under this assumption by MOSES (Fig. 15) is reached at $1.06 \mathrm{~mA}$ in the vertical plane and at $1.15 \mathrm{~mA}$ in the horizontal plane. It should be kept in mind that the threshold values can sensitively vary if another parametrization of the bunch length as a function of the current is chosen or if the product of bunch length and synchrotron tune is not constant as assumed.

\section{INCOHERENT TUNE SHIFTS}

Despite the fact that the quadrupolar wakes do not contribute to the mode coupling, they nevertheless give rise to an incoherent tune shift, which can be calculated by using the same formulas as a corresponding coherent tune shift with $\omega_{s}$ as the synchrotron angular frequency, $e$ as elementary charge, and $E$ as energy of the beam, i.e.,

$$
\frac{\Delta \nu}{\Delta I \nu_{s}}=\frac{e}{4 \sqrt{\pi} \omega_{s} \sigma_{\tau} E} \sum_{i} \beta_{i} \operatorname{Im}\left(Z_{\perp i}^{\mathrm{eff}}\right)=\frac{e}{2 \omega_{s} E} \sum_{i} \beta_{i} \kappa_{\perp i},
$$
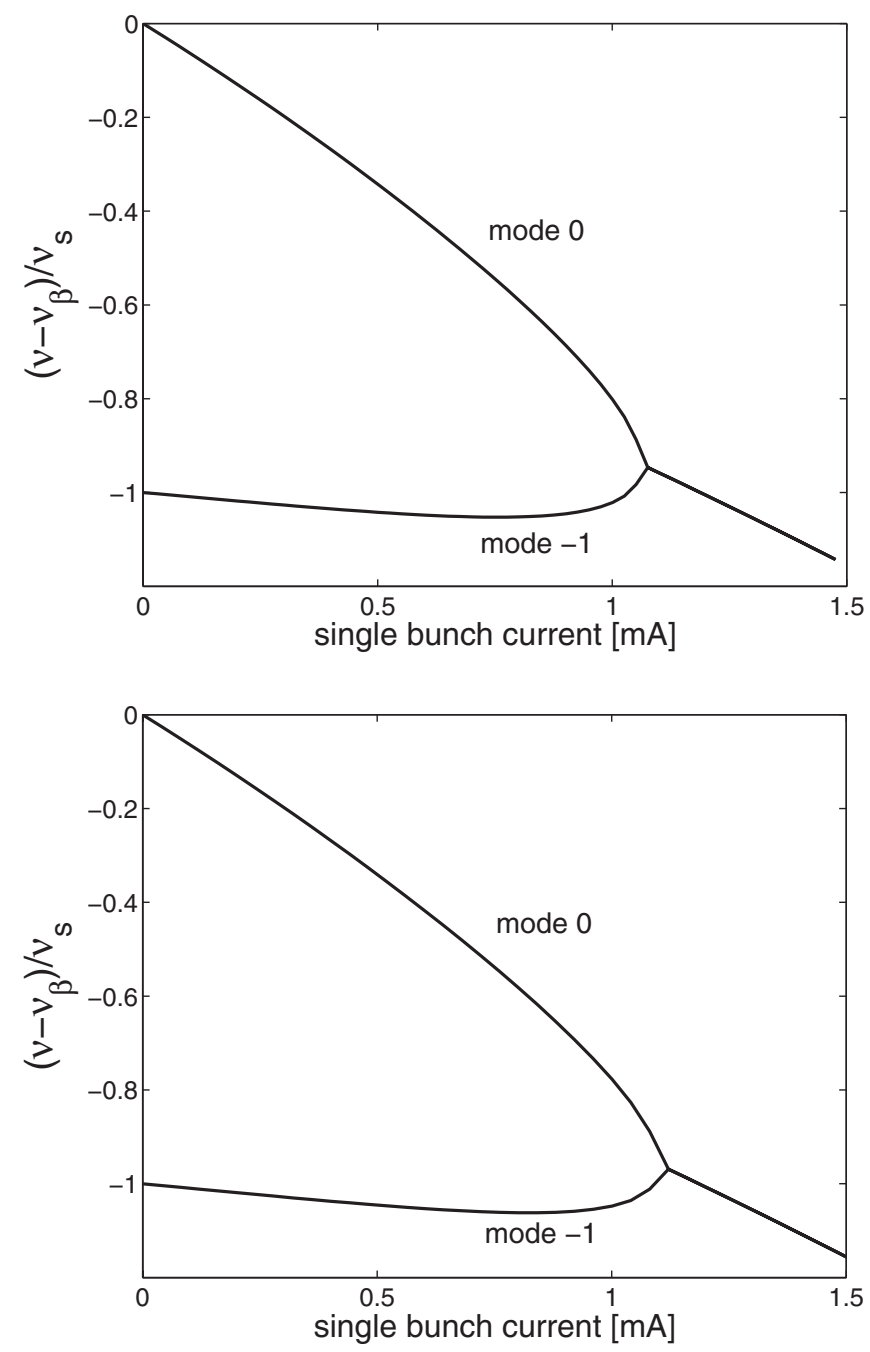

FIG. 15. The vertical (above) and horizontal (below) mode detuning obtained from the geometrical and resistive wall impedance using MOSES. Normalization of the betatron tune shifts on the current dependent incoherent synchrotron tune $\nu_{s}(I)$. The TMC instability occurs at a slightly higher current above the mode merging when the growth rate of the instable mode is larger than the radiation damping.

where $i$ enumerates the different elements and $\kappa_{\perp}$ represents the transverse kick factor:

$$
\kappa_{\perp}=\frac{1}{\pi} \int_{0}^{\infty}|\tilde{\rho}(\omega)|^{2} \operatorname{Im} Z_{\perp}(\omega) d \omega,
$$

where $\tilde{\rho}(\omega)$ represents the Fourier transformation of the bunch distribution in time domain. The chosen normalization of the tune shift on the current dependent synchrotron tune in the equation has the advantage that it makes the factor before the summation of the $\beta$-weighted effective impedance constant according to the explanation in the last section. In the next section, the current dependent synchrotron tune $\nu_{s}$ will be replaced by the zero current synchrotron tune $\nu_{s 0}$ to make comparison to measured data 


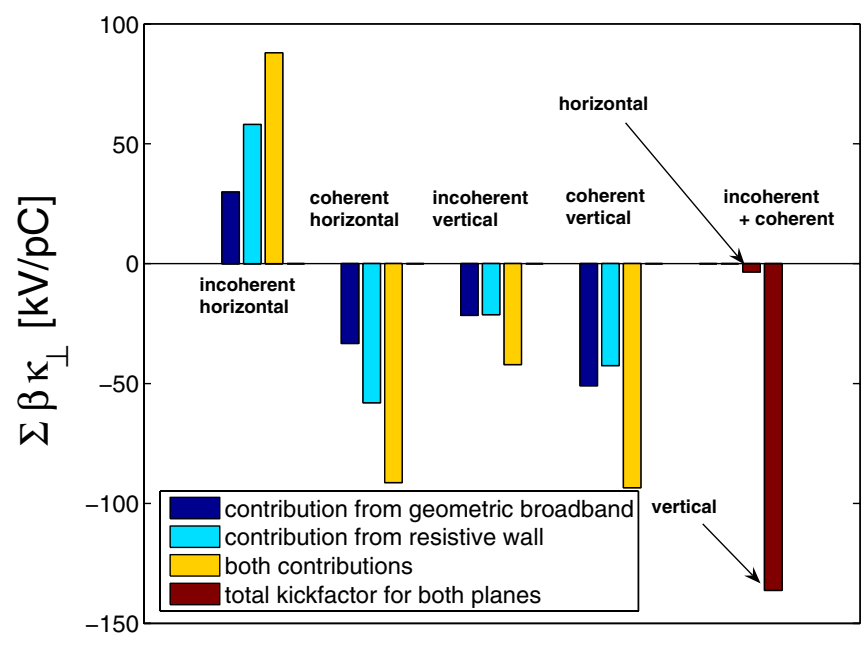

FIG. 16. (Color) The different contributions to the $\beta$-weighted vertical and horizontal kick factors (synonym to the tune shifts apart from a constant factor) at a bunch length of $5 \mathrm{~mm}$.

possible. Consequently, the independence of the factor on current is lost, whereas the independence on the rf voltage remains. The horizontal, vertical coherent, and incoherent tune shifts were calculated either by using $Z_{H}, Z_{V}$, or $Z^{\text {quad }}$ as $Z_{\perp}$ in the kick factor definition. Figure 16 shows that the incoherent horizontal tune shift is opposite to the coherent one which leads to a compensation of both. However, the vertical incoherent and coherent tune shift add up positively. The $\beta$-weighted geometrical and resistive wall impedance contribute almost equally to the total $\beta$-weighted vertical kick factor, whereas the $\beta$-weighted resistive wall impedance contributes twice more to the $\beta$-weighted horizontal kick factor than the $\beta$-weighted geometrical impedance.

\section{COMPARISON OF THE CALCULATED MODE DETUNING TO MEASUREMENTS}

The complete mode detuning vs current is obtained by adding the incoherent tune shifts to the coherent ones. This changes the slopes of the coherent mode detuning, but not the thresholds (Fig. 15). In addition, the slopes of the mode detuning are also changed by a different normalization of the tune shifts, namely, henceforth on the zero current synchrotron tune $\nu_{s 0}$. The calculated detuning curves are compared to measurements taken with the tune monitor. In order to make the coherent beam modes visible, a very slight positive chromaticity was applied and the beam was excited by a monochromatic shaker whose frequency was swept over the observed frequency range. The peaks displayed by the tune monitor were attributed to the different coherent modes and the measured frequencies of the peaks normalized on the synchrotron frequency measured at a current close to zero. However, in order to measure the values of the threshold current, the beam was injected at zero chromaticity without external excitation until beam blowup and current saturation occurred.

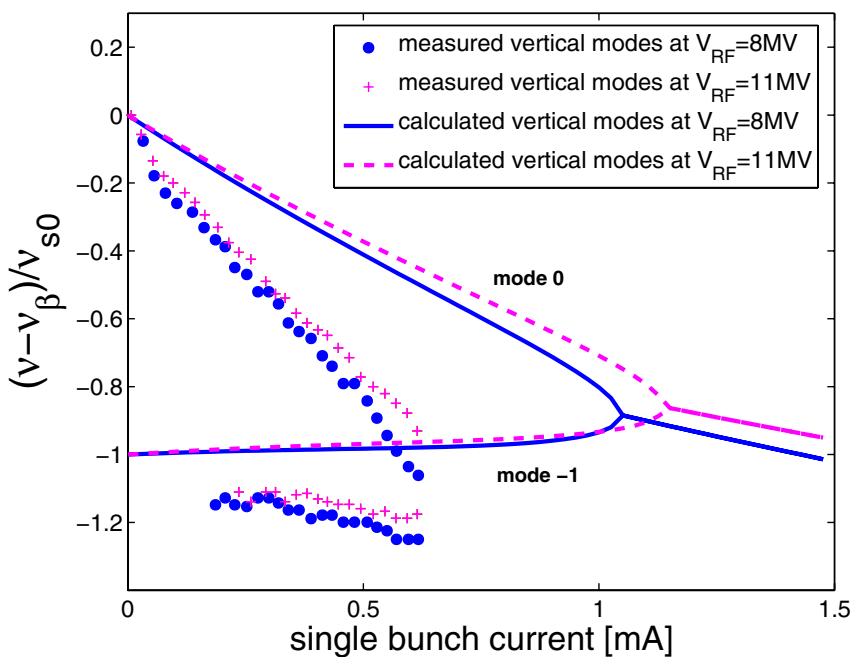

FIG. 17. (Color) Measured and calculated vertical detuning versus single bunch current at $8 \mathrm{MV}$ and $11 \mathrm{MV}$ rf voltage, measured thresholds slightly below $0.7 \mathrm{~mA}$. Normalization of the betatron tune shifts on the zero current synchrotron tune $\nu_{s 0}$.

\section{A. Vertical plane}

The calculated vertical modes 0 and -1 have a weaker detuning and couple at a higher current than the measured detuning (Fig. 17). The variation of the rf voltage is expected only to change the effective impedance in Eq. (25) through the change of the bunch length. Therefore the variation of the tune shift at different $\mathrm{rf}$ voltages provides some information of the impedance spectrum. At varying rf voltage the calculated modes in the vertical plane behave in the same way as the measured data (Fig. 17). The spectral representation of the vertical impedance modeling seems to therefore be correct. After the replacement of a NEG-coated $\mathrm{Cu}$-covered stainless steel chamber by a NEG-coated aluminum chamber of $5 \mathrm{~m}$ length and $8 \mathrm{~mm}$ aperture, the slope of the measured vertical mode detuning was only slightly weaker and threshold current only slightly higher.

\section{B. Horizontal plane}

The measured independence of mode 0 on increasing current and on varying rf voltage is due to the compensation of the coherent and incoherent tune shift. This effect is in good agreement with the prediction. However, the computed slope of mode 1 is weaker than the measured slope. The poor reproduction is possibly linked to an imperfect parametrization of the bunch length and incoherent synchrotron tune as a function of current (see Sec. V). The measured data of mode -1 are less certain because during the measurements the mode was difficult to recognize at nearly zero chromaticity. So the reproduction of this mode through the model data is only poor (Fig. 18).

However, when a NEG-coated $\mathrm{Cu}$-covered stainless steel chamber of $8 \mathrm{~mm}$ gap was exchanged for a NEGcoated aluminum chamber a large change occurred. The 


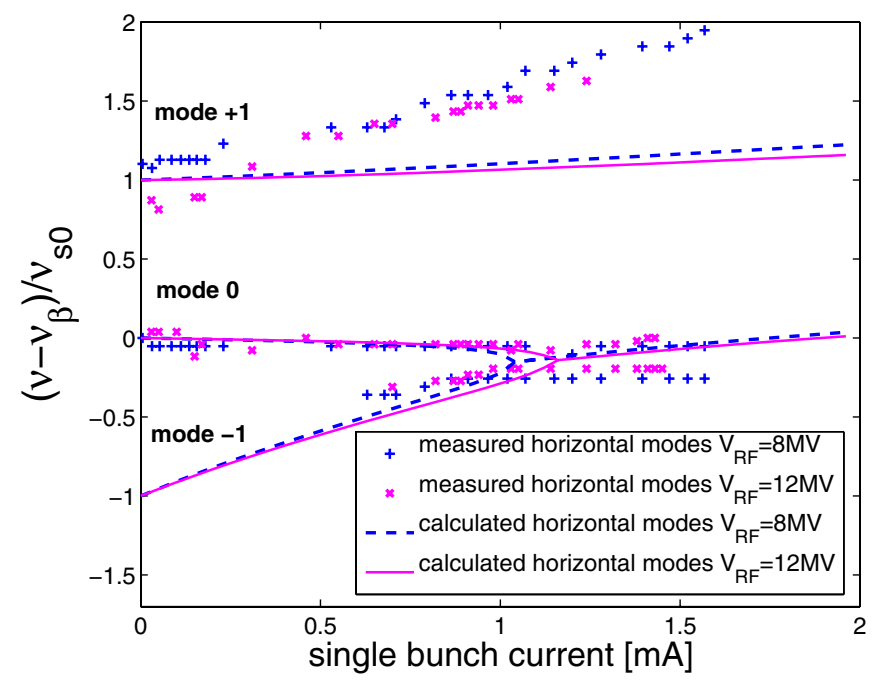

FIG. 18. (Color) Measured and calculated horizontal detuning versus single bunch current at 8 and $12 \mathrm{MV}$ rf voltage. Measured thresholds around $1.3 \mathrm{~mA}$. Normalization of the betatron tune shifts on the zero current synchrotron tune $\nu_{s 0}$.

threshold originally measured at $1.3 \mathrm{~mA}$ moved to $1.7 \mathrm{~mA}$ despite the fact that the corresponding change in the model was only small. Measurement of the transverse impedance of this type of chamber using the bump method [14] had already revealed that the NEG-coated Cu-covered stainless steel chambers present an abnormally high impedance which cannot be explained by the model presented in this paper. In fact after the replacement of this chamber the obtained discrepancy between the measured data and the impedance model reveals that the $\beta$-weighted horizontal impedance predicted by the model seems to be too large compared to the measurements. Furthermore, recent measurements showed that, in the horizontal plane, the increase of the rf voltage decreases the value of the horizontal threshold contrary to the behavior on the vertical plane. So the vertical effective impedance decreases with smaller bunch length, whereas the horizontal effective impedance increases with smaller bunch length. A possible explanation for this is the different positions of the resonances in both impedance spectra. The vertical impedance spectrum is dominated by a resonance of about $2 \mathrm{GHz}$, whereas most resonances of the horizontal spectrum are at a much higher frequency ( $\geq 6.6 \mathrm{GHz}$ BPM resonance). For a resonance at high frequency $\omega_{r} \sigma_{\tau} \gg 1$ the effective impedance decreases with increasing bunch length $\sigma_{\tau}$ (neglecting for the time being the resistive wall impedance):

$$
Z_{\perp}^{\mathrm{eff}}\left(\sigma_{\tau}\right)=\mathrm{const}\left[1+\frac{1}{2\left(\omega_{r} \sigma_{\tau}\right)^{2}}\left(1-\frac{1}{Q^{2}}\right)\right]
$$

$Q$ is the quality factor of the resonance. However, the condition $\omega_{r} \sigma_{\tau} \gg 1$ would be only correctly fulfilled if the frequency of the BPM's principal resonance were at a higher value. For an improved modeling the resistive wall would have to be less pronounced and the resonances would have to be at higher frequency than in the actual model.

\section{CONCLUSION}

The developed impedance model based on a distinct transverse impedance evaluation on the vertical and horizontal plane is in rather good agreement with the measured mode detuning on both planes. This achievement was possible through the identification of the role of the quadrupolar part of the wake potential induced by the flat geometry of the vacuum chambers. The sensitivity of the horizontal single bunch threshold on vacuum chamber changes, in particular, in low-gap sections could be well explained by the developed model. A simple analysis, with the impedance calculated assuming the same offset for the leading and trailing particle, i.e. without decomposition into dipolar and quadrupolar parts, would have resulted in a very high current threshold of the horizontal TMC instability. This is in contradiction with our measurements which show similar current thresholds in both planes. The understanding of this effect has been given in this paper as a cancellation (addition) of the dipolar and the quadrupolar wake in the horizontal (vertical) plane. One important consequence is that any change of the vertical profile of the chambers does not only generate impedance in the vertical plane, but also in the horizontal plane. At the ESRF, the large horizontal $\beta$-function in many ID sections has further amplified the effect to a point that the gradual replacement of 19 and $15 \mathrm{~mm}$ chambers by $10 \mathrm{~mm}$ chambers has caused a stronger reduction of the TMC instability current threshold in the horizontal plane than in the vertical plane. This study also showed that the ESRF storage ring is very sensitive to impedance related effects because of the high modularity of its vacuum system and has potential of improvement.

Nevertheless the model is still incomplete. The computed vertical TMCI threshold is too high compared to the measured one whereas the computed horizontal TMCI threshold is too low. If for instance Landau damping due to betatron tune spread intervenes, the value of the TMCI threshold can no longer be taken as a pure measure of the transverse impedance. The horizontal plane could particularly be concerned. Therefore the beam dynamics has still to be better understood by the realization of further measurements as well as by a better model also including the betatron tune spread caused by the quadrupolar wake.

\section{ACKNOWLEDGMENTS}

The author would like to thank P. Elleaume for his support and his corrections. The author thanks R. Nagaoka for many fruitful discussions. Furthermore, the author thanks J.L. Revol for his support in taking measurements and numerous discussions. He thanks 
L. Farvacque and T. Perron for their help and useful comments. He also thanks B. Plan and J. Pasquaud for the provision of many drawings of vacuum chamber elements. Finally, the author thanks W. Bruns for his good support in using his program.

[1] R. Nagaoka, Impact of Resistive Wall Wakefields Generated by Low-Gap Chambers on the Beam at the ESRF, PAC 2001, Chicago, pp. 3531-3533.

[2] W. Bruns, Electromagnetic Field Solver GDFIDL, http:// www.gdfidl.de

[3] R. Nagaoka, J.L. Revol, and P. Kuske, Incoherent Transverse Tune Shifts due to Resistive Low-Gap Chambers, EPAC 2002, Paris, pp. 1541-1543.

[4] S. Heifets, A. Wagner, and B. Zotter, SLAC/AP110, 1998.

[5] A. Chao, S. Heifets, and B. Zotter, Phys. Rev. ST Accel. Beams 5, 111001 (2002).

[6] A. Burov and V. Danilov, Phys. Rev. Lett. 82, 2286 (1999).

[7] K. Yokoya, Part. Accel. 41, 221 (1993).

[8] A. Chao, Physics of Collective Beam Instabilities in High Energy Accelerators (Wiley, New York, 1993).

[9] In the most general case, the transverse wake vector has a tensorial dependence on the offset vector of the exciting particle. Consequently, also a term $B(s) x y_{0}$ would be possible. However, typically the cross dependence of a transverse wake component on the other coordinate of the offset is very weak and can be neglected. Furthermore, other combinations of $W_{l}(s, x, y)$ with $y_{0}$ either have no effect on the transverse wakes or are of higher order and can be neglected.
[10] W. K. H. Panofsky and W. A. Wenzel, Rev. Sci. Instrum. 27, 967 (1956).

[11] R. L. Gluckstern, J. van Zeijts, and B. Zotter, Phys. Rev. E47, 656 (1993).

[12] Y. Shobuda and K. Yokoya, Phys. Rev. E 66, 056501 (2002).

[13] R. Nagaoka, Study of Resistive Wall Effects on SOLEIL, EPAC 2004, Lucern, pp. 2038-2040.

[14] T. Perron, L. Farvacque, and E. Plouviez, Vertical Effective Impedance Mapping of the ESRF Storage Ring, EPAC 2004, pp. 2053-2055.

[15] S. S. Kurennoy, Using a Ceramic Chamber in Kicker Magnets, PAC 1993, Washington D.C., p. 3420.

[16] Y.H. Chin, CERN SL/92-49 (AP), Geneva, 1992.

[17] The MAFIA Collaboration, Users' guide MAFIA, CST.

[18] R. Nagaoka, A Brief Summary on the Preliminary Testing of GDFIDL and Comparisons with MAFIA, Soleil Internal Note, 2002.

[19] K. Nakamura, The Broadband Impedance of the Spring-8 Storage Ring, EPAC 1996, Barcelona, pp. 1099-1101.

[20] It should be mentioned that, adding the monopolar wake of this geometry to the dipolar wake, the value would be about 12 times larger. But this value is still small.

[21] Y.H. Chin, MOSES, CERN/LEP-TH/88-5

[22] K. Scheidt, Dual Streak Camera at the ESRF, EPAC 1996, Barcelona, pp. 1624-1626.

[23] H. Wiedemann, Particle Accelerator Physics (Springer, New York, 1998), 2nd ed., Vol. 2, p. 385.

[24] It was checked that at the energy of the ESRF-ring of $6 \mathrm{GeV}$ the intrabeam scattering does not affect this relation. 